\title{
Transmitted duplication of 8p23.1-8p23.2 associated with speech delay, autism and learning difficulties
}

\author{
Mary Glancy*,1, Angela Barnicoat ${ }^{2}$, Rajan Vijeratnam ${ }^{3}$, Sharon de Souza ${ }^{4}$, Joanne Gilmore ${ }^{1}$, \\ Shuwen Huang ${ }^{5}$, Viv K Maloney ${ }^{5}$, N Simon Thomas ${ }^{6}$, David J Bunyan ${ }^{5}$, Ann Jackson ${ }^{1}$ and \\ John CK Barber ${ }^{5,6,7}$
}

\begin{abstract}
${ }^{1}$ NE London Regional Cytogenetics Laboratory, Great Ormond Street Hospital NHS Trust, London, UK; ${ }^{2}$ NE Thames Regional Clinical Genetics Service, Institute of Child Health, London, UK; ${ }^{3}$ Cedar House, St Michael's Centre for Primary Care, Middlesex, UK; ${ }^{4}$ Paediatric Department, Chase Farm Hospital, Enfield, Middlesex, UK; ${ }^{5}$ National Genetics Reference Laboratory (Wessex), Salisbury NHS Foundation Trust, Salisbury, Wiltshire, UK; ${ }^{6}$ Wessex Regional Genetics Laboratory, Salisbury NHS Foundation Trust, Salisbury, Wiltshire, UK; ${ }^{7}$ Human Genetics Division, Southampton University School of Medicine, Southampton General Hospital, Southampton, UK
\end{abstract}

Duplications of distal $8 p$ with and without significant clinical phenotypes have been reported and are often associated with an unusual degree of structural complexity. Here, we present a duplication of 8p23.18p23.2 ascertained in a child with speech delay and a diagnosis of ICD-10 autism. The same duplication was found in his mother who had epilepsy and learning problems. A combination of cytogenetic, FISH, microsatellite, MLPA and oaCGH analysis was used to show that the duplication extended over a minimum of $6.8 \mathrm{Mb}$ between 3539893 and $10323426 \mathrm{bp}$. This interval contains 32 novel and 41 known genes, of which only microcephalin (MCPH1) is a plausible candidate gene for autism at present. The distal breakpoint of the duplicated region interrupts the CSMD1 gene in 8p23.2 and the medial breakpoint lies between the MSRA and RP1L1 genes in 8p23.1.

An interchromosomal insertion between a normal and polymorphically inverted chromosome 8 is proposed to explain the origin of this duplication. Further mapped imbalances of distal $8 p$ are needed to determine whether the autistic component of the phenotype in this family results from the cumulative imbalance of many genes or dosage imbalance of an individual susceptibility gene.

European Journal of Human Genetics (2009) 17, 37-43; doi:10.1038/ejhg.2008.133; published online 20 August 2008

Keywords: autism; epilepsy; duplication; 8p23.1-8p23.2; array $\mathrm{CGH}$; $M C P H 1$

\section{Introduction}

Chromosome 8 is an average chromosome with respect to length, gene content, repeat content and degree of segmental duplication. ${ }^{1}$ However, the final $15 \mathrm{Mb}$ of the short

*Correspondence: M Glancy, North East London Regional Cytogenetics Laboratory, Great Ormond Street Hospital NHS Trust, 2nd Floor, Institute of Neurology, Queen Square House, Queen Square, London, WC1N 3BG, UK. Tel: + 44207829 8870; Fax: + 44207813 8578;

E-mail: glancm@gosh.nhs.uk

Received 28 August 2007; revised 24 April 2008; accepted 20 June 2008; published online 20 August 2008 arm is unusual in the variety of relatively complex chromosome anomalies that arise within it, $^{2-6}$ the degree of copy number polymorphism associated with the defensin gene clusters $^{7-9}$ and the degree of positive selection affecting the defensin and microcephalin (MCPH1) genes in humans. ${ }^{1}$

Clinically significant duplications of distal $8 \mathrm{p}$ can be distinguished from euchromatic variants ${ }^{5}$ but are still rare, and overlapping duplications are associated with marked variation in phenotype from severe ${ }^{10}$ to mild $^{5,6,11}$ to unaffected. $^{12,13}$ Here, we present a new family with an interstitial duplication of distal $8 \mathrm{p}$ and a syndrome that 
includes autism in a child and epilepsy in the mother. The duplication has been mapped with fluorescence in situ hybridisation (FISH), microsatellites, multiplex ligationdependent probe amplification (MLPA) and oligonucleotide array comparative genomic hybridisation (oaCGH). We briefly review overlapping duplications and the possible candidate genes for the autistic component of the phenotype.

\section{Methods}

\section{Molecular cytogenetics}

G-banded chromosomes were analysed at the 550 or higher band level and FISH carried out with 12 Ensembl tiling path (www.ensembl.org/homo_sapiens/cytoview) BACs, which map from bands 8 p23.3 to 8p21.2 (Table 1). Genes assigned to the duplicated region were obtained from the Ensembl database (v32) using the MartView data export tool (www.ensembl.org/Multi/martview).

\section{Molecular genetics}

DNA was extracted from whole blood using a salt precipitation technique. Fluorescent PCR amplification was carried out using standard conditions. Microsatellites were selected from within the duplicated interval using Ensembl and the Genome Database (www.gdb.org; Table 1). One primer from each pair was fluorescently labelled

Table 1 BAC FISH, MLPA and oligonucleotide array results in mother and son

\begin{tabular}{|c|c|c|c|}
\hline${ }^{a}$ Band & $\begin{array}{l}{ }^{b} B A C / \text { microsatellite* } \\
M L P A^{* *} / \text { array CGH loci*** }\end{array}$ & $\begin{array}{l}\text { Start and stop position } \\
\text { (Mb from telomere) }\end{array}$ & ${ }^{c}$ Results \\
\hline $8 p 23.3$ & 338B22 & $467644-668409$ & Normal \\
\hline $8 p 23.3$ & D8S504* & $1004963-1005161$ & $136 ; 138$ \\
\hline $8 p 23.3$ & D8S264* & $2117752-2117882$ & $123 ; 127$ \\
\hline $8 p 23.2$ & CSMD1 end & 2782789 & - \\
\hline $8 p 23.2$ & $336 N 16$ & $2888584-3078882$ & Normal \\
\hline $8 p 23.2$ & Oligo*** & $3313384-3313443$ & Normal \\
\hline $8 p 23.2$ & D8S1824* & $3539893-3540122$ & $227 ; 240 ; 246$ \\
\hline $8 p 23.2$ & Oligo*** & $3848594-3848653$ & Duplicated \\
\hline $8 p 23.2$ & $16 \mathrm{H} 11$ & $4177855-4396741$ & Duplicated \\
\hline $8 p 23.2$ & D8S518* & $4475013-4475263$ & $242 ; 244 ; 247$ \\
\hline $8 p 23.2$ & CSMD1 start & 4839736 & - \\
\hline $8 p 23.2$ & RP5-991O23 & $5316020-5469277$ & Duplicated \\
\hline $8 p 23.1$ & D8S1742* & $6201415-6201550$ & $128 ; 136 ; 140$ \\
\hline $8 p 23.1$ & CTD-2629I16 & $6674740-6695317$ & Duplicated \\
\hline $8 p 23.1$ & $D E F B 1^{* *}$ & $6715511-6722939$ & Duplicated \\
\hline REPD & $122 \mathrm{~N} 11$ & $7367548-7578862$ & Three signals \\
\hline $8 p 23.1$ & $211 C 9$ & $8479797-8687720$ & Duplicated \\
\hline $8 p 23.1$ & MFHAS1 (MASL1)** & $8680942-8787978$ & Duplicated \\
\hline $8 p 23.1$ & PPP1R3B & $9032916-9045616$ & Duplicated \\
\hline $8 p 23.1$ & D8S503* & $9270573-9270784$ & $244 ; 246 ; 250$ \\
\hline $8 p 23.1$ & $M S R A^{* *}$ & $9949240-10323808$ & Duplicated \\
\hline $8 p 23.1$ & $112 \mathrm{G} 9$ & $10028624-10236504$ & Duplicated \\
\hline $8 p 23.1$ & Oligo $* * *$ & $10323367-10323426$ & Duplicated \\
\hline $8 p 23.1$ & Oligo*** & $10511353-10511405$ & Normal \\
\hline $8 p 23.1$ & D8S520* & $10593772-10593964$ & $188 ; 190$ \\
\hline $8 p 23.1$ & Oligo*** & 10660074 & Normal \\
\hline $8 p 23.1$ & D8S550* & $10918965-10919233$ & $256 ; 268$ \\
\hline $8 p 23.1$ & GATA4** & $11599162-11654918$ & Normal \\
\hline $8 p 23.1$ & $589 N 15$ & $11626380-11804128$ & Normal \\
\hline REPP & - & - & - \\
\hline $8 p 23.1$ & D8S552* & $12786525-12786692$ & $166 ; 174$ \\
\hline $8 p 22$ & $433 L 7$ & $14277096-14462154$ & Normal \\
\hline $8 p 22$ & D8S1731* & $15282675-15282909$ & $219 ; 233$ \\
\hline $8 p 22$ & 809L8 & $18239259-18460085$ & Normal \\
\hline $8 p 21.3$ & CGAT1** & $19305952-19584552$ & Normal \\
\hline $8 p 21.3$ & D8S1786* & $22489342-22489551$ & $207 ; 209$ \\
\hline $8 p 21.3$ & D8S1771* & $25497152-25497377$ & $227 ; 229$ \\
\hline $8 p 21.2$ & 14117 & $26205457-26377530$ & Normal \\
\hline $8 q 24.3$ & 4811 & $145496869-145668487$ & Normal \\
\hline
\end{tabular}

Grey shading indicates the extent of duplication; Italics used for CSMD1 start and endpoints.

${ }^{a} \mathrm{G}$-dark bands in bold.

bACs RP11 unless indicated.

'Numbers indicate the size of the microsatellite alleles found at informative loci. 
and the products analysed on an ABI 3100 automated sequencer.

MLPA was carried out using the standard protocol of MRC-Holland. Three additional in-house MLPA probes corresponding to the three genes on chromosome 8 (MFHAS1 (MASL1), DEFB1 and GATA4 - sequences are available upon request) were combined with MRC-Holland probe set P036, which contains five chromosome 8 probes (MFHAS1, PPP1R3B, MSRA, GATA4 and CGAT1).

\section{Oligonucleotide array CGH}

OaCGH was carried out using test genomic DNAs obtained using a standard salt extraction method and reference normal human male genomic DNA (Promega Corporation G147A 19813601, Madison, WI). DNA quantity and quality were assessed by UV/Vis spectrophotometry using the ND-1000 Spectrophotometer (NanoDrop Technologies, Rockland, DE) and gel analysis on a 0.8\% agarose gel. The Agilent 44K Human Genome CGH microarray (G4410B, Batch number 0000013686, Agilent Technologies) consists of $\sim 4300060$-mer oligonucleotide probes with an average spatial resolution of $\sim 35 \mathrm{~kb}$ and was applied to detect genome-wide copy number changes. In brief, patient and reference DNAs were digested with AluI and RsaI and the restriction enzymes were inactivated by incubation at $65^{\circ} \mathrm{C}$. The Cy5- and Cy3-labelled DNA sample pair were combined and mixed with human Cot-1 DNA. Before hybridisation, the samples were heated at $95^{\circ} \mathrm{C}$ for $3 \mathrm{~min}$ and then incubated for $30 \mathrm{~min}$ at $37^{\circ} \mathrm{C}$. Labelled target solution was hybridised to the $4 \times 44 \mathrm{~K}$ array with three other samples using SureHub chambers (G2534A) in a $65^{\circ} \mathrm{C}$ rotisserie oven (G2545A) set to rotate at 20 r.p.m. for $24 \mathrm{~h}$. After hybridisation, the microarray slide was washed and dried according to the Agilent oligonucleotide arraybased CGH for genomic DNA analysis protocol version 4.0 (G4140-90010). The microarray slide was scanned immediately using an Agilent microarray scanner (G2565BA) and image and data analyses were performed using the Agilent CGHAnalytics (v3.3) microarray software.

\section{Clinical histories}

The proband is the first-born child of unrelated parents of Argentinian origin. He was born after an otherwise uncomplicated pregnancy by normal delivery at term with a birth weight of $3.36 \mathrm{~kg}$. Concerns about his development arose from around 2 years of age because of delayed speech. There had been mild delay in motor milestones with independent walking achieved at around 1.5 years. When assessed at the age of 3.5 years, he had around five single words in both English and Spanish but was described as having receptive language skills at a higher level. There was no jargon or echolalia or joint attention. He tended to play alone in nursery, but had no repetitive play. He would hand flap when excited and was upset by changes in routine. Eye-to-eye contact was poor and he demonstrated no spontaneous affection. He was diagnosed with autism satisfying the ICD-10 criteria. He had no seizures.

In addition to his developmental problems, there were complaints of poor feeding and diarrhoea. He required antitubercular treatment after developing hilar shadowing and a lower lobe collapse with a positive Mantoux test.

His development was monitored and had shown slow progress with persistent autistic features and continuing severe language delay. Examination showed a well-nourished non-dysmorphic boy; specifically, there were no features suggestive of Kabuki syndrome. His head circumference was above the 50th centile and height and weight on the 50th centile.

The proband's mother was aged 23 years at the time of his birth. She was diagnosed with epilepsy and learning problems. The degree of her difficulty was difficult to ascertain given her inability to speak English. She was nondysmorphic on limited physical examination. She was prescribed two anticonvulsants before pregnancy, although she had discontinued them during pregnancy. Further details were uncertain; in addition, she was treated for tuberculosis during the pregnancy. The proband's father, aged 33 years at the time of his birth, had been diagnosed with psychiatric problems and had a previous history of substance abuse; details of his medical condition and treatment are unknown.

The couple have a second son 3 years younger than the proband. At the age of 1.5 years, this boy has mild expressive language delay but no features in the autistic spectrum. He is non-dysmorphic and has normal growth parameters.

\section{Results}

G-banded analysis showed a duplication of the distal short arm of chromosome 8 in the proband and his mother (Figure 1A-B) and normal chromosomes in the proband's father and younger brother. Using dual colour FISH in both mother and son, five BACs from bands 8p23.1 and 8p23.2 were duplicated (Figure $2 \mathrm{a}-\mathrm{d}$ ) and BAC RP11-122N11 gave three signals (Figure $2 \mathrm{~b}$; Table 1 ). All other probes gave normal results (Table 1). FISH and molecular genetic analysis, using microsatellite and MLPA probes, indicated that the duplication of $8 \mathrm{p} 23.1-8 \mathrm{p} 23.2$ includes a minimum of $\sim 6.7 \mathrm{Mb}$ between 3539893 (D8S1824) in 8p23.2 and 10236504 bp (112G9) in 8p23.1 (Table 1 based on Ensembl v36/7).

OaCGH was used to look for further possible imbalances in $8 \mathrm{p}$ or at other sites across the genome and confirmed a duplication with a minimum size of $6.47 \mathrm{Mb}$ between 3848594 and 10323426 bp (Ensembl v36/7; Figure 2e; Table 1). The array result narrowed the distal duplication breakpoint to $226 \mathrm{~kb}$ between the duplicated microsatellite locus D8S1824 at $3539893 \mathrm{bp}$ and the first normal oligonucleotide clone distal to the duplication at 
$3313443 \mathrm{bp}$. This interval extends from introns 7 to 11 of CSMD1 and must therefore interrupt this gene. The array results place the medial 8 p23.1 breakpoint within the $188 \mathrm{~kb}$ between the last duplicated oligonucleotide at $10323426 \mathrm{bp}$ and the first normal oligonucleotide at $10511353 \mathrm{bp}$. When the array and informative microsatellite results are combined, the duplication is a minimum of $6.78 \mathrm{Mb}$ in size between 3539893 and $10323426 \mathrm{bp}$ and a maximum of $7.19 \mathrm{Mb}$ between 3313443 and 10511353 bp (Table 1).

Two further possible small areas of duplication with $\log 2$ ratios of 0.38 were identified; 16 clones covering $231 \mathrm{~kb}$ between 145464363 and 145695514 bp in 8q24.3 and 11 clones covering $194 \mathrm{~kb}$ between 48448631 and 48431311 bp in 3p21.31. A duplication within 8q24.3 was not confirmed using BAC RP11-48I1 and there were no suitable FISH clones with which to test the $3 p$ region, which corresponds to the Plexin-B1 precursor gene that has no known pathology (OMIM 601053).

The duplication is thought to be insertional because of the closeness of the G-dark duplicated band $8 \mathrm{p} 23.2$ to G-dark 8p22 (Figure 1A-B), the three signals from REPD BAC RP11-122N11 (Figure 2b and d), the very distal location of the single-copy BAC $589 \mathrm{~N} 15$ on the duplicated chromosome (Figure 2d), and the three alleles that were seen with five independent microsatellite markers (Table 1).

The karyotype of the proband can therefore be written as: 46,XY,dir dup ins(8)(p23.1;p23.1p23.2)mat.ish dir dup ins(8) (pter->p23.1::p23.2->p23.1::p23.1-> qter) (RP11-338B22+, $\mathrm{RP} 11-336 \mathrm{~N} 16+, \mathrm{RP} 11-16 \mathrm{H} 11+$,RP5-991O23 + ,CTD-2629I16+ , $\mathrm{RP} 11-122 \mathrm{~N} 11+, \mathrm{RP} 11-589 \mathrm{~N} 15+, \mathrm{RP} 11-112 \mathrm{G} 9+, \mathrm{RP} 11-211 \mathrm{C} 9+$, $\mathrm{RP} 11-16 \mathrm{H} 11+, \mathrm{,RP} 5-991 \mathrm{O} 23+, \mathrm{CTD}-2629 \mathrm{I1}+, \mathrm{,RP} 11-122 \mathrm{~N} 11+$, $\mathrm{RP} 11-211 \mathrm{C} 9+, \mathrm{RP} 11-112 \mathrm{G} 9+, \mathrm{RP} 11-122 \mathrm{~N} 11+, \mathrm{RP} 11-433 \mathrm{~L} 7+$,
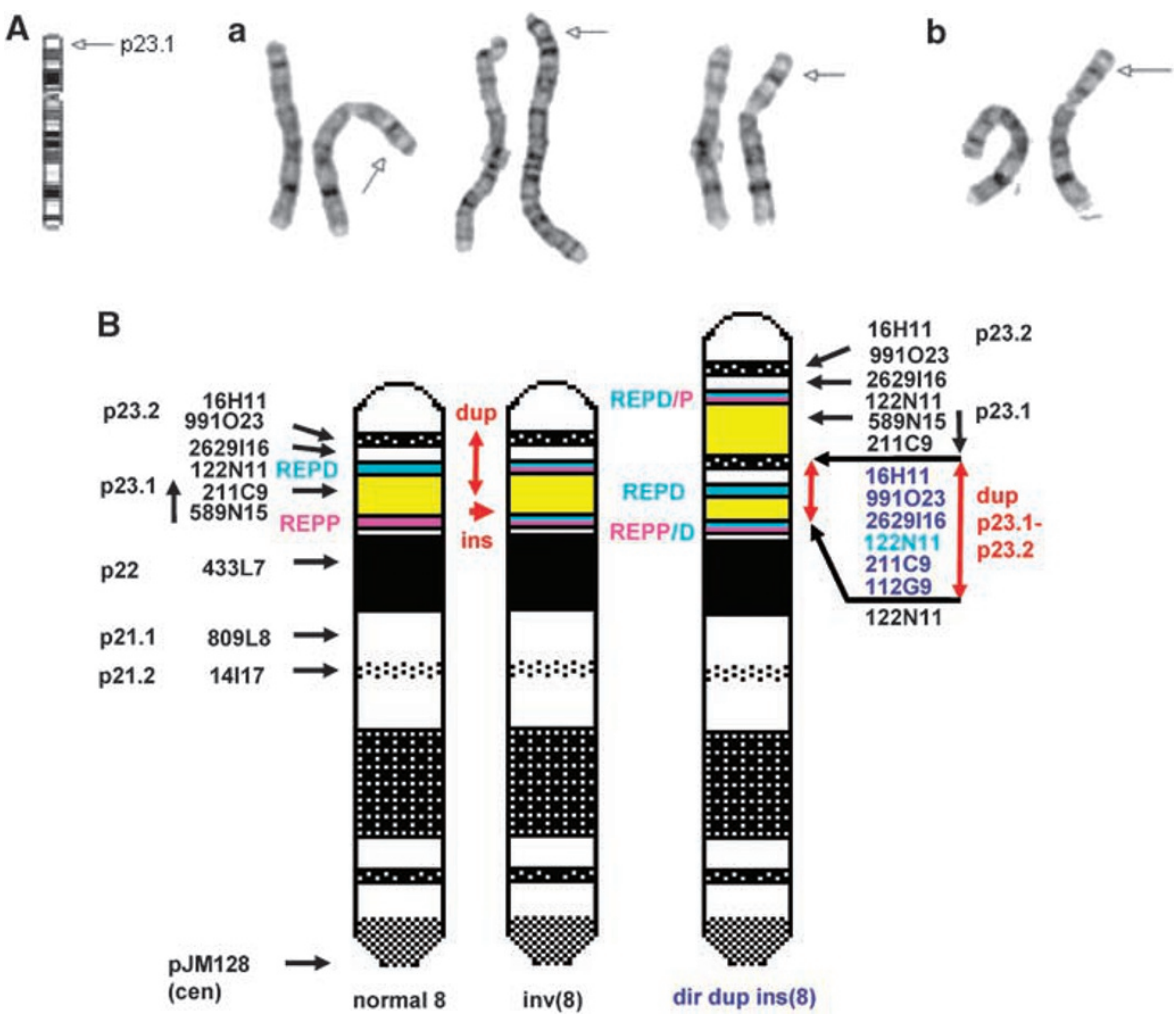

Figure 1 (A and B) Partial karyotypes of (a) the proband and (b) his mother. Note the increased interval between bands p22 and p23.2 and the additional G-dark in the proximal part of the increased interval (arrowed on the right hand duplicated chromosome in each case). (B) Idiogram of the proposed insertional duplication of distal 8p23.1-8p23.2 from a normal chromosome 8 into a polymorphically inverted chromosome 8 adjacent to REPP/D in proximal 8p23.1. On the left hand normal chromosome, distal 8 p23.1 is in white, REPD is in blue, REPP is in purple, the interval between them in yellow and proximal 8p23.1 in white again. The double-headed vertical red arrow indicates the extent of the duplication on the normal chromosome and the vertical black upward arrow next to BACs RP11-211C9 and RP11-589N15 indicates the 'genome browser' orientation of the REPP to REPD interval. In the central chromosome, REPP and REPD are shown in both colours as the result of the common polymorphic inversion and the horizontal single-headed red arrow indicates the proposed point of insertion adjacent to the composite REPD/P repeat. The duplicated chromosome is on the right with the vertical double-headed red arrows showing the position and extent of the duplication. Duplicated BACs are shown in blue and the BAC, which gave three signals (RP11-122N11) is in turquoise. Note the orientation of the duplicated BACs is the same as that on the left hand normal chromosome. By contrast, the reverse order of BACs RP11-589N15 and RP11-211C9 is indicated by the downward vertical black arrow. 

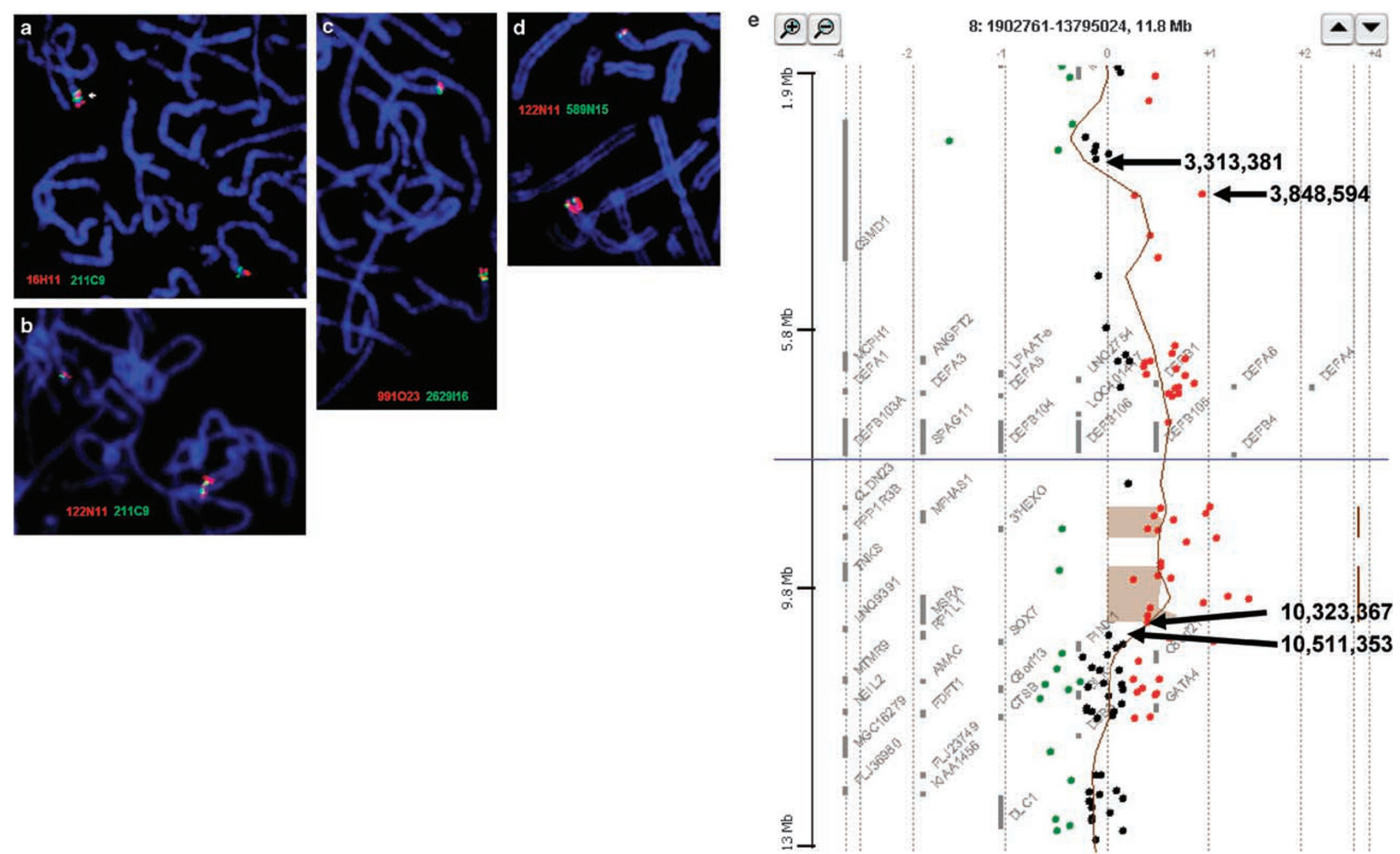

Figure 2 (a-d) Representative dual colour FISH in metaphases from the proband and mother; (a) direct duplication of both RP11-16H11 (red) from 8p23.2 and RP11-211C9 from 8p23.1 (green) in the proband; (b) the duplication of RP11-211C9 (green) alternating between three distinct RP11-122N11 signals (red) in a higher resolution cell in the mother; (c) direct duplication of both RP5-991023 (red) from 8p23.2 and CTD-2629116 from 8p23.1 (green) in the mother; (d) the single-copy RP11-589N15 signals (green) located very distally within the block of signals from RP11-122N11 in the mother. (e) Agilent 44K oligonucleotide array result from the proband analysed with Analytics v3.3 software. The black arrows point to the normal and duplicated clones that flank the duplication with the base pairs given for the p-telomeric edge of each oligonucleotide probe (see also Table 1 ). 
RP11-809L8 + ,RP11-14I17 +, RP11-48I1+).arr cgh dup(8) (p23.1p23.2)(B35:CHR8:3,848,594->10,323,426+ +).

\section{Discussion}

This family has a directly transmitted duplication of 8p23.1-p23.2 with a minimum size of $6.78 \mathrm{Mb}$ extending from 3539893 to 10323426 bp (shaded area Table 1) and a phenotype that includes speech delay, autism, epilepsy and learning difficulties. This is apparently a unique rearrangement as we are not aware of a direct precedent for this duplication in the literature and there was no evidence for predisposing repeats at the duplication breakpoints of the kind associated with other recurrent complex rearrangements of $8 \mathrm{p} .^{2,3,6,10}$

The medial 8p23.1 breakpoint of the inserted region is unusual in that it occurs between rather than within the olfactory receptor repeats (REPD and REPP) that map to both ends of band 8p23.1 and mediate other 8p rearrangements. ${ }^{2}$ The duplication is direct (Figure 2a) but is thought to be insertional because:

1. The duplicated part of G-dark $8 p 23.2$ is quite close to band $8 \mathrm{p} 22$ in the partial G-banded karyotypes (Figure 1A) and therefore difficult to reconcile with a simple direct duplication.

2. There are three signals from the REPD BAC RP11122N11 (Figure 2b), which contains variable defensin gene cluster sequences. The most distal and proximal signals are located on the chromosome, which received the insertion. The signal between them is located on the inserted segment. The possible triplication of the variable defensin domain was not confirmed by oaCGH but this is likely to have been a reflection of the use of control DNA of unknown defensin copy number.

3. BAC RP11-589N15 is normally proximal to BACRP11211C9 but was clearly distal to the duplication and very close to the most distal signal from REPD BAC RP11122N11 (Figure 2d). This suggests that the duplicated segment was inserted into a chromosome with the polymorphic inversion of 8 p23.1 in which the order of RP11-211C9 and RP11-589N15 was reversed (Figure 1B).

4. Three alleles were seen with five independent microsatellite markers (Table 1).

We also considered that the duplication might have been inserted into one of the repeats but this would have resulted in four signals - one from the repeat unaffected by the insertion, one from the repeat contained within the duplication and two signals from the repeat split by the insertion. This was not observed and our best interpretation was therefore an insertion adjacent to a composite REPP/D as illustrated in Figure 1B.

The 8p23.1 breakpoint and duplication in this family creates an additional truncated copy of the CUB and sushi multiple domains gene CSMD1 (OMIM 608397) from the $5^{\prime}$ prime end to a breakpoint between intron 7 at $3313384 \mathrm{bp}$ and intron 11 at 3539893 bp in band 8p23.2. However, this is unlikely to be pathogenic as two copies of the normal gene remain and copy number variation including truncated copies of this gene have been reported extending to $1.3 \mathrm{Mb}$ (L1013 in the Database of Genomic Variants) ${ }^{14}$ and $2.3 \mathrm{Mb}$ in size (cnp676). ${ }^{15}$ The medial 8p23.1 breakpoint of the inserted segment, lies between the duplicated peptide methionine sulfoxide reductase A (MSRA) and the retinitis pigmentosa 1-like protein 1 (RP1L1) gene outside the duplication. In animal models, the overexpression of $M S R A$ is protective against oxidative damage (OMIM 601250) and no pathology has yet been associated with RPL1L1 (OMIM 608581). The insertion site is adjacent and proximal to the proximal REPP/D in the inverted chromosome. Thus, the breakpoints do not harbour strong candidates for the phenotype in this family.

The duplication in this family includes 41 known and 32 novel genes. However, as many as 22 of the known genes are members of the $\alpha$-and $\beta$-defensin cluster regions, which vary in copy number in the normal population ${ }^{7,9}$ and are therefore unlikely to be responsible for the phenotype. Of the remaining 19 genes, few are obvious candidates for the phenotype in this family. However, the 8p23.1 duplication syndrome mediated by REPP and REPD is associated with mild dysmorphism, speech delay and learning difficulties

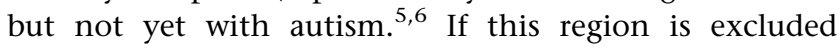
together with the defensin gene clusters, only five known genes remain (CSMD1, MCPH1, ANGPT2, AGPAT5 and NP_997295) of which only MCPH1 has an OMIM morbid entry. Recessive mutations and deletions of this gene (OMIM 251200, 606858 and 607117) are associated with microcephaly, mental retardation and premature chromosome condensation ${ }^{16}$ and additional copies of this gene have been associated with the increased head size found in two previously reported patients with $8 \mathrm{p}$ duplications and triplications by Giorda et al. ${ }^{10}$ As there is increasing evidence of an association between rapid head growth in the first year of life and subsequent autism, ${ }^{17,18}$ it is conceivable that the duplication of this gene could give rise to autism or less severe psychomotor developmental problems in patients with additional copies of MCPH1.

Other duplications of 8 p23.1 have been reported with a wide variety of presentations, including developmental delay, heart disease and autism but their gene content has not been determined yet. ${ }^{19,20}$ A cytogenetic duplication of 8 p21 to 8 p23 was also associated with autism ${ }^{21}$ but, like the well-known inverted duplications of $8 \mathrm{p}$ (inv dup del(8)s), includes a large more proximal segment of $8 \mathrm{p}$, which contains other candidate genes for syndromic autism.

Our results add to the extreme heterogeneity found in both syndromic and non-syndromic autism using array CGH. ${ }^{22-24}$ In particular, Jacquemont et al. ${ }^{22}$ found nine different imbalances in 29 patients with syndromic autism, 
only one of which corresponded to a known autism susceptibility locus. In addition, Sebat et al. ${ }^{23}$ found distinct gains and losses in another 17 patients but none of these 26 imbalances or de novo copy number variants involved 8p. However, the recently established Autism Chromosome Rearrangement database contains a variety of cytogenetic rearrangements that suggest that the distal $8 \mathrm{p}$ may yet harbour one or more autism susceptibility loci. $^{24}$

In conclusion, we report a duplication of $8 \mathrm{p} 23.1$ to 8p23.2 associated with speech delay, autism, epilepsy and learning difficulties. At the current level of knowledge, only MCPH1 is a plausible candidate for the autistic component of the phenotype in this family. Thus, further mapped imbalances of distal $8 \mathrm{p}$ are needed to provide better prognostic information for patients in the future and to investigate whether the autism results from the cumulative imbalance of many of the genes involved or dosage imbalance of an individual susceptibility gene.

\section{URLs}

Ensembl tiling path (http://www.ensembl.org/homo_ sapiens/cytoview)

MartView data export tool (www.ensembl.org/Multi/ martview).

OMIM (http://www.ncbi.nlm.nih.gov/entrez/query)

Database of Genomic Variants (http://projects.tcag.ca/ variation/)

The Autism Chromosome Rearrangement Database (http://projects.tcag.ca/autism/)

\section{Acknowledgements}

We are grateful to the Sanger Centre for providing the Ensembl tiling path clones and to Dr Hiroaki Shizuya for kindly providing BAC 51D11. SH and VKM are supported as part of the National Genetics Reference Laboratory (Wessex) by the UK Department of Health Genetics, Embryology and Assisted Conception Unit. The image enhancement equipment used for this work was provided by the Welcome Trust and Trust Funds of Salisbury NHS Health Care Trust

\section{References}

1 Nusbaum C, Mikkelsen TS, Zody MC et al: DNA sequence and analysis of human chromosome 8. Nature 2006; 439: 331-335.

2 Giglio S, Broman KW, Matsumoto N et al: Olfactory receptor-gene clusters, genomic-inversion polymorphisms, and common chromosome rearrangements. Am J Hum Genet 2001; 68: 874-883.

3 Giglio S, Calvari V, Gregato G et al: Heterozygous submicroscopic inversions involving olfactory receptor-gene clusters mediate the recurrent $\mathrm{t}(4 ; 8)(\mathrm{p} 16 ; \mathrm{p} 23)$ translocation. Am J Hum Genet 2002; 71: $276-285$.
4 Sugawara H, Harada N, Ida T et al: Complex low-copy repeats associated with a common polymorphic inversion at human chromosome 8p23. Genomics 2003; 82: 238-244.

5 Barber JCK, Maloney V, Hollox EJ et al: Duplications and copy number variants of $8 \mathrm{p} 23.1$ are cytogenetically indistinguishable but distinct at the molecular level. Eur J Hum Genet 2005; 13: $1131-1136$

6 Barber JCK, Maloney VK, Huang S et al: 8p23.1 duplication syndrome; a novel genomic condition with unexpected complexity revealed by array CGH. Eur J Hum Genet 2008; 16: 18-27.

7 Hollox EJ, Armour JAL, Barber JCK: Extensive normal copy number variation of a $\beta$-defensin antimicrobial gene cluster. Am J Hum Genet 2003; 73: 591-600.

8 Taudien S, Galgoczy P, Huse K et al: Polymorphic segmental duplications at 8p23.1 challenge the determination of individual defensin gene repertoires and the assembly of a contiguous human reference sequence. BMC Genomics 2004; 5: 92-103.

9 Aldred PM, Hollox EJ, Armour JAL: Copy number polymorphism and expression level variation of the human alpha-defensin genes DEFA1 and DEFA3. Hum Mol Genet 2005; 14: 2045-2052.

10 Giorda R, Ciccone R, Gimelli G et al: Two classes of low-copy repeats co-mediate a new recurrent rearrangement consisting of duplication at $8 \mathrm{p} 23.1$ and triplication at 8p23.2. Hum Mutat 2007; 28: 459-468.

11 Brooks SS, Genovese M, Gu H, Duncan CJ, Shanske A, Jenkins EC: Normal adaptive function with learning disability in duplication 8p including p22. Am J Med Genet 1998; 78: 114-117.

12 Engelen JJM, Moog U, Evers JLH, Dassen H, Albrechts JCM, Hamers AJH: Duplication of chromosome region 8p23.1-> p23.3: a benign variant? Am J Med Genet 2000; 91: 18-21.

13 Harada N, Takano J, Kondoh T et al: Duplication of 8p23.2 a benign: cytogenetic variant? Am J Med Genet 2002; 111: 285-288.

14 Sebat J, Lakshmi B, Troge J et al: Large-scale copy number polymorphism in the human genome. Science 2004; 305: 525-528.

15 Redon R, Ishikawa S, Fitch KR et al: Global variation in copy number in the human genome. Nature 2006; 444: 444-454.

16 Trimborn M, Bell SM, Felix C et al: Mutations in microcephalin cause aberrant regulation of chromosome condensation. Am J Hum Genet 2004; 75: 261-266.

17 Courchesne E, Carper R, Akshoomoff N: Evidence of brain overgrowth in the first year of life in autism. JAMA 2003; 290: 337-344.

18 Dawson G, Munson J, Webb SJ, Nalty T, Abbott R, Toth K: Rate of head growth decelerates and symptoms worsen in the second year of life in autism. Biol Psychiatry 2007; 61: 458-464.

19 Tsai C-H, Graw SL, McGavran L: 8p23 duplication reconsidered: is it a true euchromatic variant with no clinical manifestation? J Med Genet 2002; 39: 769-774.

20 Kennedy SJ, Teebi AS, Adatia I, Teshima I: Inherited duplication, $\operatorname{dup}(8)(\mathrm{p} 23.1 \mathrm{p} 23.1) \mathrm{pat}$, in a father and daughter with congenital heart defects. Am J Med Genet 2001; 104: 79-80 (letter).

21 Papanikolaou K, Paliokosta E, Gyftodimou J et al: A case of partial trisomy of chromosome 8p associated with autism. J Autism Dev Disord 2006; 36: 705-709.

22 Jacquemont ML, Sanlaville D, Redon R et al: Array-based comparative genomic hybridisation identifies high frequency of cryptic chromosomal rearrangements in patients with syndromic autism spectrum disorders. J Med Genet 2006; 43: 843-849.

23 Sebat J, Lakshmi B, Malhotra D et al: Strong association of de novo copy number mutations with autism. Science 2007; 316: 445-449.

24 Autism Genome Consortium, Szatmari P, Paterson AD, Zwaigenbaum L et al: Mapping autism risk loci using genetic linkage and chromosomal rearrangements. Nat Genet 2007; 39: 319-328. 